\title{
Research and Application Analysis of Feature Binding Mechanism
}

\author{
Youzhen $\operatorname{Han}^{1}$ and Shifei Ding ${ }^{1,2}$ \\ ${ }^{1}$ School of Computer Science and Technology, \\ China University of Mining and Technology, \\ 221116, Xuzhou, Jiangsu, China \\ 15152114529@126. com \\ ${ }^{2}$ Key Laboratory of Intelligence Information Processing, \\ Institute of Computer Technology, Academy of Sciences, \\ 100190, Beijing City, Beijing, China \\ dingsf@cumt.edu.cn
}

\begin{abstract}
In order to recognize the object of the external world, brain need to integrate the information of different cortical areas, then form a complete world. And binding problem study on a process to percept a complete object by integrating information, which scattering on different cortical areas. As a central problem of cognitive science and neuroscience, the concept of feature binding is becoming to the focus of consciousness argument. At the beginning of this paper, we introduced the concept, characteristics and theory source of feature binding. And according to the main theoretical research methods of this mechanism, combining the latest advance at home and abroad, we made a systematical review of the research situation of feature binding problem applying in perceptual learning. At last, we pointed out the key point of the further study, which may be refined research of bundled brain mechanism on different cognition process and systematical study of general bundled brain mechanism.
\end{abstract}

Keywords: feature binding, vision perception, perceptual learning, synchronous neural oscillations, $\gamma$-band oscillations, cognitive neuroscience.

\section{Introduction}

The so-called binding problem is to study a way, integrating the information scattering on different cortical areas to percept a complete object. This theory supports rich theoretical and experimental bases for the research of image understanding based on visual hierarchical perceptual mechanism. Thereby, the research on the cognitive process and practical application of feature binding mechanism has an important theoretical breakthrough and application value. This paper attempts to outline the principle theoretical bases, and make a more systematic review about the research on the binding problem and the functional relations of cognition. We hope to raise the domestic scholars' concern in this field. 
The organizational structure of this paper is as follows: after outlined the basic theory of feature binding; we introduced the origin and progress of binding mechanism, and proposed the further research direction, in section 2 ; in section 3 , we introduced the current main theory, including the feature-integration theory, neural synchronous activation theory and bundled neural network model; section 4 mainly introduced the crucial effect of binding mechanism in the cognition process, concretely include distinguish perception, the selection of perceptual information, the memory and consciousness wakeup etc.; at last, in conjunction with our own experience in the research, we made a prospect of the progress of binding mechanism.

\section{Research Situation of Binding Mechanism}

There is a long history of the exploiting of cognition; our ancestors already have thinking about the origin and nature of humans' recognition, long time ago. Limited by the level of science, the knowledge of brain is mainly stay on the observation and guesses, lacking the study of the brain's internal theory. Until 20th century, with the breakthrough of neuron anatomy, neurophysiology and electrophysiological processes of neuron, people have a deeper understand of the structure and the basic unit of brain.In 1999, Riesenhuber and Poggi(Riesenhuber and Poggio 1999) proposed a standard quatitative model based on visual perception mechanism and visual feature computering, their theory has the error-tolerant about the location, size and shape, this give people a quantitative mathematical understanding of this bio-inspired model. Shi Zhongzhi et al., based on PCNN, proposed a computering model of feature bindingBayesian Linking Field model, research on the feature binding in visual perception. Since the PCNN extremely close to the process method which used by biological visual cortex, many scholars improved PCNN itself and have imposed a lot of modified PCNN model (Zhan et al. 2009, Huang and Jing 2007) through analyzing this model, and made it performing excellent properties.

\section{$3 \quad$ Main Approaches of Feature Binding}

There are many theories about binding mechanism. Inside, the feature-integration theory of cognitive psychology, synchronous neuronal activation theory which influential in neuroscience and also called temporal synchronization theory, and recent years proposed bundled neural network model are some most typical theories.

\subsection{Feature-Integration Theory}

Treisman's Feature-integration theory tries to use selective attention mechanism of visual space - the attention window to explain the feature binding problem(Bouvier and Treisman 2010, Di Lollo 2012). The theory considers the features from the parallel processing of early stage to the late integration are realized through the spatial attention model. The model includes a position main-map and a set of independent feature-maps. The position main-map is used for registering the location of the object, but not understands the features of that location. The feature maps mainly include two types of 
information: one is the "flag", which is used to mark whether the feature is existed in somewhere of the scope; the other is about some implicit information in the feature space arrangement. Every feature detector in feature map connected to a unit of main map. After established the unified object representation, the stored template can be matched and recognized, and the related behavior can also be completed.With further research, people realized that there are three deficiencies in the traditional feature integration theory : (1) a traditional feature binding is measured through conscious report, there is no use of unconscious implicit indicators; (2) all are inspected according to the results of immediate report, there is no inspection based on the results of the memory report; (3) the information involved only biting characteristics and location, did not involve the bundle between behavior and perception. These studies are more significance to the general bundling mechanisms.

\subsection{Synchronous Neuronal Activation Theory}

Synchronous neural activation theory (temporal synchronization) is currently considered the most influential binding theories. This theory is that, feature binding based on the synchronization activates neurons, realization through the synchronization activation of neural activity(Singer and Gray 1995, Von Der Malsburg 1994). The neurons which are characterization of the same object or trajectory can activate the corresponding behavior in time synchronization to the millisecond. And this synchronous activation is not generated in charge of different cells or between cells. This instant synchronous activation is the key of binding mechanism. Synchronous activation can make selective mark to the neuronal that are responsible for encoding, and distinguish from other objects' activated neuronal responses. This highly selective temporal structure that allows different cell populations in the same neural networks activates synchronously and differentiates from each other. In addition, time-based synchronization binding also serves as a mechanism of selecting next processing object. Because of accurate synchronous activation, the specific content become a desired activation result, and make other brain neurons perceive such a result. These selectively activated neurons will complete binding operations in different regions, and make this activation model in the neural network get the further process.

This theory is that the binding is accomplished by the synchronization of neural activity(Singer and Gray 1995, Von Der Malsburg 1994). The neural basis of time synchronization is the connection of the cells which react to the features stimulating in senses at the same time. The features may be bound quickly by the connection through the accurate millisecond time synchronization and spatially parallel manner. This mechanism has the advantages of low-performance requirements to the functional structure, high flexibility and adapt to a wide range.

\subsection{The Neural Network Model of Binding}

The neural network model of binding was developed by Watanabe, Nakanishi and Aihara(Watanabe et al. 2001). The model is composed of a primary map (primary map) and two advanced modules. Two modules are responsible for two different characteristics in the primary map, and each module is divided into three different 
levels, between all levels are pair-wise two-way connected. The external representation of the object, it is represented by the "overall dynamic cell collection" which are distributed in the primary map and two advanced modules. The details of the information are encoded in the main map, and rough, complex feature information is characterized in the two advanced modules. Feature binding is completed in this 'overall dynamic cell clusters'.

The bases of the model are "functional links" and "two-way link" of neural networks. Functional link is dynamic link between specific neurons which use coupled sensor encoding neural network's time pulse (temporal spike). This link is different from traditional synaptic connections for its value can be changed with the excited level of neural network dynamics change, its intensity is decided by the time consistency level of occasional action potentials and each neuron, directly affected by the spatial and temporal dynamics. These cells are called the 'dynamic cell clusters' that are linked by the functional link. The model uses two-way link, it can explain time synchronization nerve release phenomena without introducing neural oscillation unit of the time synchronization theory.

\section{The Functions of Binding Mechanism in the Perceptual Processes}

As for the specific function of binding mechanism of perceptual processes, different researchers come to different conclusions. As Friedman-Hill found that right parietal is related with visual features binding in the perception report(Friedman-Hill et al. 2003); while Ashbridge found that right parietal and binding have no direct relationship in the visual search task, related with the selection of spatial position only, the ventral temporal cortex is responsible for the feature binding(Ashbridge et al. 1999); And numerous studies show that the hippocampus is related with features binding in memory(Mitchell et al. 2000); Prabhakaran, etc. observed in working memory storage consolidation feature, prefrontal got activation(Prabhakaran et al. 2000); Llinas etc. found that nonspecific thalamic circuits is responsible for perceiving a wake-up binding(Llinás 1988). In addition, in the specific binding task, the left middle temporal lobe , cerebellum, left frontal lobe, the parietal lobe, left medial frontal gyrus, right anterior cingulate gyrus, right frontal gyrus, right prefrontal, anterior cingulate cortex, left center back and other brain regions can be activated. It can be seen in different cognitive tasks clearly, brain mechanism of binding is extremely complex.

\subsection{Binding and Perception Distinction}

In the neurophysiologic level, it was found binding is related with the situational perception distinction height; external sensory input formed structural characterization under the function of binding. There is evidence that nerve released simultaneously depends on the presentation of stimulation. Spatially separate cells shown strong synchronization only in response to the same object. If the two cell populations made reaction to the two independent objects which were moving in the different directions, then these two cell populations' activation almost had no time relation(Castelo-Branco 
et al. 2000). These results indicate that each object corresponds to its own synchronous activation mode,different objects have different neural activation patterns. Therefore, it can achieve different objects' perception distinction based on synchronous neural release binding mechanism. Tallon-Baudry through EEG studies has found that the perception of the object's consistency is associated with a specific $\gamma$-band activity(Tallon-Baudry et al. 1996). This relationship between high-frequency activity and the perception of the object's consistency also confirmed by a lot of other paradigms studies. Since $\gamma$-band activities reflect the information of time synchronization, it also reflect binding mechanisms' role situation.

\subsection{Binding and the Selection of Perception Information}

Fries record the cat's visual cortex neural responses under the conditions of binocular rivalry(Fries et al. 1997). They give two stimulations that moved in different directions to the wakefulness cats' left and right eye at the same time. According to the direction of eye movement to speculate perception advantage, making the grating deviation as identification of the eye movement direction perceives the selection result. Studies found that neuronal activity stimulated a high degree of synchronization, these neuronal activity are used to characterize the perception; while nerve cells that used to process the ignored stimulation exhibit only weak time relationship. Meanwhile, the simultaneously released of the recording point is accompanied with the advantaged $\gamma$ oscillations, characterized the selected stimulated neurons, and the intensity of the $\gamma$-band increased, but the neurons which make a reaction to the neglect stimulation, its strength decreased. The results show that, at least in the early stages of visual perception, sensory information's dynamic selection and inhibition are associated with the synchronization regulation of neural activity. Thus, this also proved that binding and the selection of perception information are closely related.

\subsection{Binding and Memory}

\subsubsection{Binding and Working Memory}

Research shows that the function of working memory depends on the nerve cells' temporal coordination on the time (temporal coordination). Tallon-Baudry etc. studied the relationship between synchronization of $\gamma$-band and working memory(Tallon-Baudry et al. 1998) They found that in a task of visual delayed matching, the accurate synchronization of ventral occipital and frontal area was strengthened. Sarnthein's etc. study found that in a visual-spatial working memory task, time consistency of forehead and posterior parietal parts' electrodes has also been strengthened. And this increase occurred in $\gamma$-band, but in the lower frequency bands (for example $\theta$ frequency) does not occur. It shows that the time binding mechanisms also plays an important role in working memory.

Recent studies of cognitive aging found that compared with the young, on the memory of binding characteristics the elderly has the obvious shortages, while on the memory of single feature there was no difference. This shows that the binding 
mechanism has a relationship with multi-feature combination's information coding, save or extract. This conclusion has also been supported by brain imaging studies. This shows that for normal subjects' binding capacity, the working memory tasks that need for feature binding can lead to greater hippocampus activation. The study not only proved the presence in working memory binding problems, but also made important contributions to reveal the neural basis of the binding.

\subsubsection{Binding and Episodic Memory}

Lukeu etc. pointed out that episodic memory depends on the ability of individual tied up the central message of an event with contextual information(Lekeu et al. 2002). Center information refers to the theme of the event (such as the main content of the conversation). Contextual information includes spatial and temporal characteristics of events, encoding mode (such as visual or auditory, etc.), physical characteristics (such as pitch or color) and emotional status. Contextual information can act as the clues to extract center information. In order to form memory traces which is complete and relevant a particular event, it is not enough to code two kinds of information independently. You must also establish a binding between central feature and contextual feature. The possibility of an event extracted depends on the number and strength of these two kinds of information bundled. It was also found that there were two forms of binding to central feature and contextual feature: Intentional and unintentional binding. These two forms of binding between different characteristics have different effects. They found that under the conditions of the unintentional binding, the left middle temporal lobe and cerebellum activated in the word recognition; the left frontal and parietal areas activated in combination recognition. Under the conditions of the intentional binding, in the word recognition, the left middle frontal gyrus, right anterior cingulate and right frontal gyrus activated; in combination recognition, the right prefrontal activated.

\subsection{Binding and Consciousness Awakening}

\subsubsection{Binding and Perception Awakening}

Some studies have found that in the awakening state and rapid eye movement sleep (REM) state, in the EEG (electroencephalogram) or MEG (magneto encephalography) appears frequencies of $\gamma$-band. In the deep sleep state such frequencies disappears. The similarity of REM state and wakefulness on the high-frequency activity indicates that in these two states, the synchronization of the neural activity frequency band is associated with the generating process of the arousal state. Another study found that, under the height anesthesia, the high frequency components of the perception awakening also disappeared. Since $\gamma$-band activity is associated with synchronous activation of nerve cells. From the above results we can speculate that the binding mechanisms involved in the process of the sense change from the depth anesthesia or deep sleep state to the awake state. 


\subsubsection{Binding and Consciousness}

At present, a main point in the study of consciousness holds that sense should be understood as a number of interacting systems' functions. These systems include sensing systems, memory structure, the executive control center and emotional and motivational systems(Engel and Singer 2001). The specific functions of consciousness mainly for two aspects(Huisong 2012): (1) cross-system coordination function, namely how a variety of different nature process integrated, and how they are in harmony and consistently organized in the distributed neural activity model; (2) dynamic selection function. Since in a given time, only a part of information can enter into the consciousness. So how consciousnesses achieve the dynamical selection to the specific content? Obviously, from the meaning of binding, these two functions of consciousness are closely related with binding mechanisms. In fact, the previously mentioned perception distinction, information selection, working memory, awakening and other mental processes are the main content of consciousness(Engel and Singer 2001). The relationship between these processes and binding mechanisms also confirmed the function of binding in the consciousness.

\section{Conclusion and Prospect}

Basing on the mechanism of feature binding, this paper systematically introduced the present study about feature binding used in perceptual learning, combining the latest research advances at home and abroad. According to our paper, the binding problem has got more and more academic attention, also is an important field in cognitive science, neuroscience and brain science. But the current research is in its infancy, there is no theory which can perfectly solve the binding problem. In addition, the brain areas which are responsible for different forms of binding may have different working mechanism in the different cognitive tasks. Therefore, with the development of neuroscience, and the increasingly deep of the research on binding problem and relationship of cognitive function. Hence force, on one hand we need to do more in-depth and meticulous research to the brain regions which are responsible for different forms of binding, on the other hand, it must strengthen systematic study of the overall brain function which is responsible for general binding problem, making connections to the variety of fragmented brain function location research, and unifying the systematic framework.

Acknowledgements. This work is supported by the National Natural Science Foundation of China (No.61379101), the National Key Basic Research Program of China (No.2013CB329502), the Basic Research Program (Natural Science Foundation) of Jiangsu Province of China (No.BK2013093), and the Students Innovation and Entrepreneurship Foundation of China University of Mining and Technology (No.201446). 


\section{References}

1. Ashbridge, E., Cowey, A., Wade, D.: Does parietal cortex contribute to feature binding? Neuropsychologia 37(9), 999-1004 (1999)

2. Bouvier, S., Treisman, A.: Feature binding signals in visual cortex. Journal of Vision 10(7), 96-96 (2010)

3. Castelo-Branco, M., Goebel, R., Neuenschwander, S., et al.: Neural synchrony correlates with surface segregation rules. Nature 405(6787), 685-689 (2000)

4. Di Lollo, V.: The feature-binding problem is an ill-posed problem. Trends in Cognitive Sciences 16(6), 317-321 (2012)

5. Engel, A.K., Singer, W.: Temporal binding and the neural correlates of sensory awareness. Trends in Cognitive Sciences 5(1), 16-25 (2001)

6. Friedman-Hill, S.R., Robertson, L.C., Desimone, R., et al.: Posterior parietal cortex and the filtering of distractors. Proceedings of the National Academy of Sciences 100(7), 4263-4268 (2003)

7. Fries, P., Roelfsema, P.R., Engel, A.K., et al.: Synchronization of oscillatory responses in visual cortex correlates with perception in interocular rivalry. Proceedings of the National Academy of Sciences 94(23), 12699-12704 (1997)

8. Huang, W., Jing, Z.: Multi-focus image fusion using pulse coupled neural network. Pattern Recognition Letters 28(9), 1123-1132 (2007)

9. Huisong, W.: affinity propagation algorithm to build context-aware learning system analysis. Fuqing Branch of Fujian Normal University (5), 46-51 (2012)

10. Lekeu, F., Van Der Linden, M., Collette, F., et al.: Effects of incidental and intentional feature binding on recognition: a behavioural and PET activation study. Neuropsychologia 40(2) (2002)

11. Llinás, R.R.: The intrinsic electrophysiological properties of mammalian neurons: insights into central nervous system function. Science 242(4886), 1654-1664 (1988)

12. Mitchell, K.J., Johnson, M.K., Raye, C.L., et al.: fMRI evidence of age-related hippocampal dysfunction in feature binding in working memory. Cognitive Brain Research 10(1), 197-206 (2000)

13. Prabhakaran, V., Narayanan, K., Zhao, Z., et al.: Integration of diverse information in working memory within the frontal lobe. Nature Neuroscience 3(1), 85-90 (2000)

14. Riesenhuber, M., Poggio, T.: Hierarchical models of object recognition in cortex. Nature Neuroscience 2(11), 1019-1025 (1999)

15. Singer, W., Gray, C.M.: Visual feature integration and the temporal correlation hypothesis. Annual Review of Neuroscience 18(1), 555-586 (1995)

16. Tallon-Baudry, C., Bertrand, O., Delpuech, C., et al.: Stimulus specificity of phase-locked and non-phase-locked $40 \mathrm{~Hz}$ visual responses in human. The Journal of Neuroscience 16(13), 4240-4249 (1996)

17. Tallon-Baudry, C., Bertrand, O., Peronnet, F., et al.: Induced $\gamma$-band activity during the delay of a visual short-term memory task in humans. The Journal of Neuroscience 18(11), 4244-4254 (1998)

18. Von Der Malsburg, C.: The correlation theory of brain function. Springer (1994)

19. Watanabe, M., Nakanishi, K., Aihara, K.: Solving the binding problem of the brain with bi-directional functional connectivity. Neural Networks 14(4), 395-406 (2001)

20. Zhan, K., Zhang, H., Ma, Y.: New spiking cortical model for invariant texture retrieval and image processing. IEEE Transactions on Neural Networks 20(12), 1980-1986 (2009) 